\title{
Experimental investigation for Mass Modification Effect in Nuclei at J-PARC
}

\section{Kawama ${ }^{* a}$}

E-mail: kawama@riken.jp

K. Aoki ${ }^{b}$, Y. Aramaki ${ }^{a}$, H. En'yo ${ }^{a}$, H. Hamagaki ${ }^{c}$, J. Kanaya ${ }^{a}$, K. Kanno ${ }^{d, a}$,

A. Kiyomichi ${ }^{e}$, Y. Komatsu ${ }^{d, a}$, S. Masumoto ${ }^{d}$, Y. Morino ${ }^{a}$, H. Murakami ${ }^{d}$,

R. Muto ${ }^{b}$, W. Nakai ${ }^{d, a}$, M. Naruki ${ }^{f}$, Y. Obara $^{d}$, K. Ozawa $^{b}$, F. Sakuma ${ }^{a}$,

S. Sawada ${ }^{b}$, M. Sekimoto $^{b}$, T. Shibukawa ${ }^{d}$, K. Shigaki $^{g}$, T. N. Takahashi $^{a}$,

Y. S. Watanabe ${ }^{d}$, S. Yokkaichi ${ }^{a}$ (J-PARC E16 Collaboration)

${ }^{a}$ RIKEN Nishina Center, RIKEN, Saitama 351-0198, Japan

${ }^{b}$ KEK, High Energy Accelerator Research Organization, Ibaraki 305-0801, Japan

${ }^{c}$ Center for Nuclear Study, Graduate School of Science, University of Tokyo, Tokyo 113-0033, Japan

${ }^{d}$ Department of Physics, University of Tokyo, Tokyo 113-0033, Japan

e Japan Synchrotron Radiation Research Institute, Hyogo 679-5198, Japan

${ }^{f}$ Department of Physics, Kyoto University, Kyoto 113-0033, Japan

${ }^{g}$ Hiroshima University, Hiroshima 739-8526, Japan

The J-PARC E16 experiment will measure the invariant mass spectra of vector mesons in the $e^{+} e^{-}$decay channel in order to investigate the mass modification of hadrons in nuclear matter. This effect was reported by a predecessor experiment, KEK-PS E325, and we plan to measure the nuclear-size and meson-momentum dependence of the modification with higher statitstics, using a $30-\mathrm{GeV}$ primary proton beam at the J-PARC high-momentum beam line. New detectors and readout circuits have been developed to cope with the high particle rate, namely, estimated as 5 $\mathrm{kHz} / \mathrm{mm}^{2}$ at most. The commissioning of the spectrometer and beam line is planned in FY2015.

XV International Conference on Hadron Spectroscopy-Hadron 2013

4-8 November 2013

Nara, Japan

\footnotetext{
* Speaker.
} 


\section{Introduction}

The origin of Hadron mass is thought to originate from the spontaneous breaking of chiral symmetry in QCD. The chiral symmetry is expected to be restored in hot and/or dense matter, thus the hadron mass in such matter is also expected to be changed. In the finite density matter, there are several theoretical predictions of hadron mass modification [1, 2, 3, 4]. One of the basic experimental method for the study of this subject is to measure the mass of vector mesons in nuclei decaying into the leptonic channel. In particular, the measurement of the low-momentum mesons is suitable because the mesons can spend a considerable time in the nuclei after production, and have a larger decay probability in the nuclei. This idea is depicted in Fig. 1, which is for the $\phi$ meson's case. In this case a proton beam is used to produce $\phi$ mesons in nuclei, and the invariant mass is obtained by measuring decayed $e^{+} e^{-}$pair. If the meson mass is modified in the nuclear matter, the shape of the obtained invariant mass could be changed. For the direct measurement of the modification of the mass spectrum shape, the leptonic decay is necessary because its possibility of the final state interaction in nuclear matter is much less than that of hadronic decay.

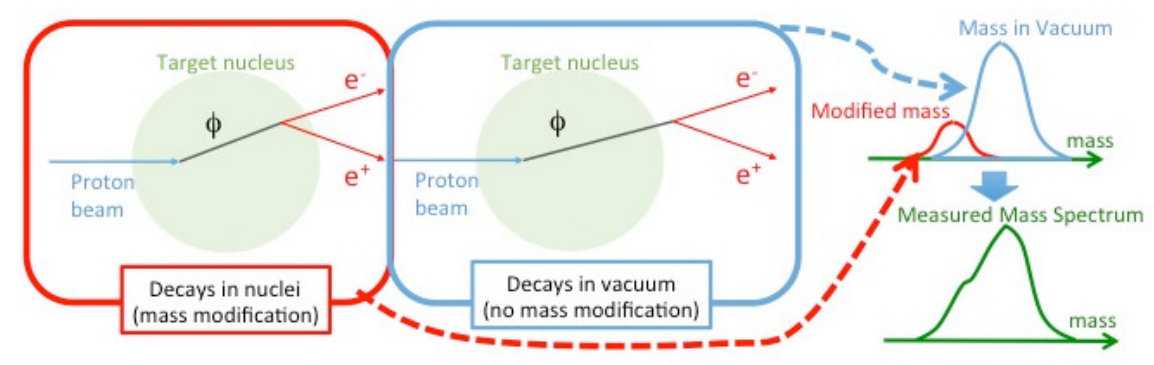

Figure 1: The basic idea of the experiment. A proton beam is used to produce vector mesons in nuclei. The produced mesons decay inside or outside nuclei, and their mass shape could be changed due to the inside-nuclear decay.

At $\mathrm{KEK}$, such an experiment using a $12-\mathrm{GeV}$ primary proton beam was performed by the KEK-PS E325 collaboration, and the mass modification of vector mesons was observed[5, 6]. Taking an advantage of the high-intensity proton beam at J-PARC, we planned a new experiment, the J-PARC E16 experiment[7, 8], for more systematic studies.

\section{Results of the predecessor experiment (KEK-PS E325)}

The $\phi \rightarrow e^{+} e^{-}$invariant mass spectrum for the $\mathrm{Cu}$ target, for the slowly-moving $\phi$ mesons $\left(\beta \gamma_{\phi}<1.25\right)$, obtained by E325[6] is shown in the left-hand panel of Fig. 2. The excess was observed on the left side of the peak, over the known mass shape, namely, the relativistic BreitWigner shape including the experimental effects in the detectors. The number of mesons in the excess can be counted by subtracting the expected mass spectrum shape and the background, and the ratio of $N_{\text {excess }} /\left(N_{\text {excess }}+N_{\phi}\right)$ was calculated for several bins of velocities and target nuclei, and summarized in the right-hand panel of Fig. 2. The lines in the figure represent the results of a model calculation[8]. The mass shift parameter $k$ is defined in the formula $m^{*} / m_{0}=1-k\left(\rho(r) / \rho_{0}\right)$, 

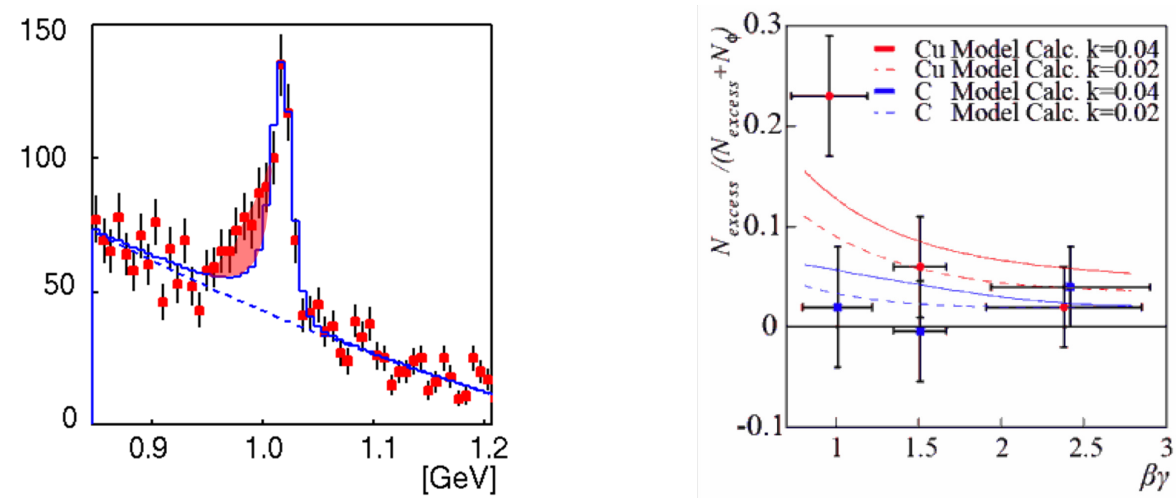

Figure 2: (left) The $\phi \rightarrow e^{+} e^{-}$invariant mass spectrum obtained in the KEK-PS E325 experiment for a $\mathrm{Cu}$ target, selecting $\beta \gamma_{\phi}<1.25[6]$. The points with error bars represent the data. The solid line is a fit result with an expected $\phi \rightarrow e^{+} e^{-}$invariant mass shape and a quadratic background (the dashed line). The hatched area represents the excess. (right) The dependence of the excess ratio, $N_{\text {excess }} /\left(N_{\text {excess }}+N_{\phi}\right)$, on the $\beta \gamma_{\phi}$ for $\mathrm{C}$ and $\mathrm{Cu}$ target data. The lines represent the results of a model calculations[8] based on the theoretical prediction with two different values of the mass shift parameter[1].

where $m^{*}$ is the modified mass in a finite density, $m_{0}$ is the mass in vacuum, $\rho_{0}$ is the normal nuclear density, and $\rho(r)$ is the nuclear matter density at the decay position of the meson. Thus $k$ means the mass-shift percentage at the normal nuclear density. The best-fit result was $k=0.034_{-0.007}^{+0.006}[6]$, while the range of $k$ was predicted as $0.01-0.04$ theoretically[1].

The result is consistent with an interpretation that the excess originates from the mesons which decayed in nuclei with the modified mass. It is essential to obtain this kind of experimental data with high accuracy for the parameter determination and comparison with theoretical predictions. For this purpose, we have proposed the J-PARC E16 experiment in order to perform systematic measurements, such as dependence on the nuclear size, meson momentum, and so on.

\section{J-PARC E16 experiment}

At J-PARC, the energy of the available primary proton beam is $30 \mathrm{GeV}$, which enlarge the production cross section of the $\phi$ meson through proton-induced nuclear reactions in comparison with the $12-\mathrm{GeV}$ beam used by KEK-PS E325. For the same detector acceptance, the yield is expected to be larger by a factor of two. Also, available beam intensity is $\sim 1 \times 10^{10}$ per spill, which is ten times larger than that used by E325. Moreover, we will construct a new spectrometer as shown in Fig. 3, and the acceptance will be enlarged by a factor of five for the $\phi \rightarrow e^{+} e^{-}$decays; therefore the $\phi$ meson yield is estimated to be 100 times as large as that of the E325 experiment.

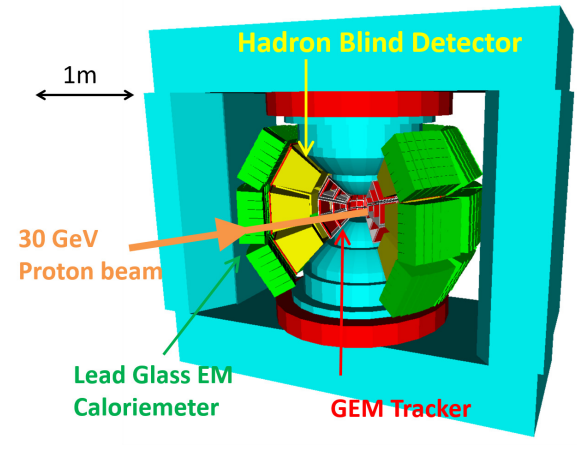

Figure 3: Schematic view of the E16 experimental setup. There are 26 detector modules surrounding the experimental target placed at the center of the magnet. Each detector module consists of the GEM tracker, HBD, and LG. 

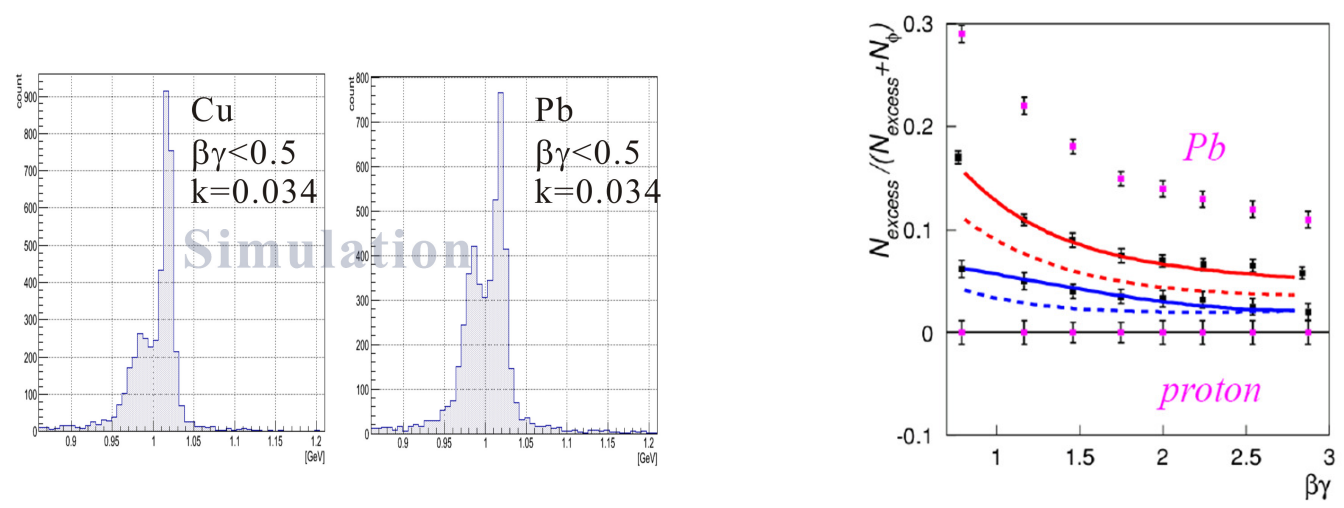

Figure 4: (left) The expected invariant mass spectra of $\phi$-meson in the E16 experiment, for $\mathrm{Cu}$ and $\mathrm{Pb}$ targets selecting $\beta \gamma_{\phi}<0.5$. The mass resolution is obtained as $5 \mathrm{MeV}$. The background is not considered. (right) The expected $\beta \gamma_{\phi}$ dependencies of the excess ratio for $\mathrm{Pb}, \mathrm{Cu}, \mathrm{C}$, and protons with the expected statistics in the E16 experiment[8]. The curves are the same as in Fig. 2.

In order to cope with the high particle rate $\left(5 \mathrm{kHz} / \mathrm{mm}^{2}\right.$ at most) due to the usage of the high-intensity primary beam, new detectors and their readout systems are required. Therefore, we have developed a tracking detector using the Gas Electron Multiplier (GEM). Achieved position resolution is $100 \mu \mathrm{m}$ [9] corresponds to the mass resolution of $5 \mathrm{MeV}$ for the $\phi$ mesons, which is two times better than that achieved by E325.

For the electron identification, hadron blind detectors (HBD)[10] and lead-glass electromagnetic calorimeters (LG) are used. The HBD also uses GEMs with the CsI photocathode to detect Cherenkov photons produced by relativistic particles in a $\mathrm{CF}_{4}$ radiator. We expect the hadron rejection powers of the HBD and LG as $1 \times 10^{-2}$ and $4 \times 10^{-2}$, respectively.

The expected invariant mass spectra in the E16 experiment are shown in the left panel of Fig. 4. These figures show results of a detector simulation using Geant4, which includes target material, detector materials and the position resolution of the GEM tracker. The selection of the slowly-moving $\phi$ mesons and the improvement in the mass resolution enhance the excess in the spectra. Also, higher-precision excess ratio data is expected to be obtained for several nuclei and various meson velocities, as shown in the right-hand panel of Fig. 4. The error bars become smaller due to the higher statistics, and the velocity bins can be divided finer than that of E325 which is shown in Fig. 2.

The high statistics data enables to reveal the momentum dependence of the mass shift and width. Figure 5 shows an expected momentum dependence of the $\phi$-meson mass in nuclei based on a theoretical curve[11]. Determination of the mass shift for

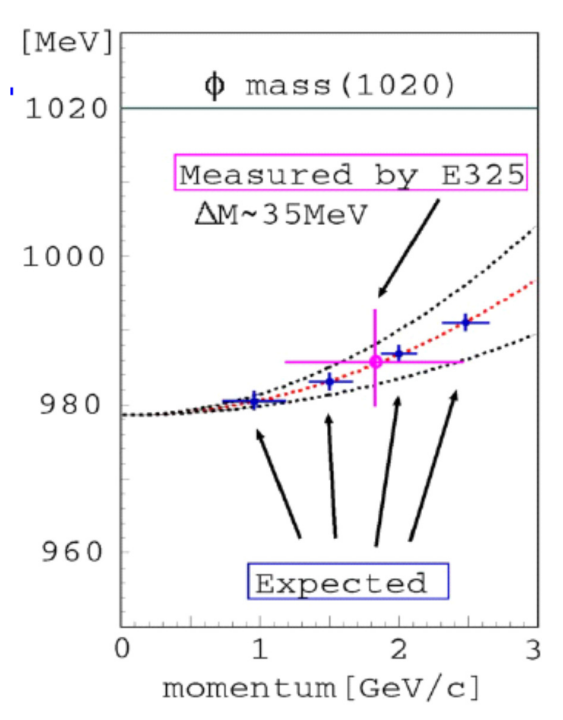

Figure 5: An expected momentum dependence of the $\phi$-meson mass in nuclei with a value measured by E325. 
each divided momentum bin could not be performed by E325 due to the limited statistics. These systematic data proves the mass modification and enables a comparison of various theoretical predictions.

\section{Summary and outlook}

The study of the hadron mass modification in nuclear matter is an unique tool to approach the origin of hadron mass. The J-PARC E16 experiment will measure the vector-meson decay in nuclei with statistics that are larger by a factor of 100 than that of the KEK-PS E325 experiment. Such high statistics data will powerfully help the systematic study of mass modification of vector meson and thus the investigation of the chiral symmetry in nuclear medium.

For the E16 experiment, the detectors and readout electronics have been developed since 2007. The mass production of a part of detectors and the construction of the J-PARC high-momentum beam line have been started in 2013. We expect to start the construction of the spectrometer magnet and installation of the detectors in FY2014. The commissioning of the beam line and the spectrometer is planned in FY2015.

\section{Acknowledgement}

We would like to give our thanks to the staffs of KEK Fuji test beam line, ELPH at Tohoku University, LEPS at SPring-8, J-PARC Hadron Experimental Facility and RIKEN RI beam factory for their help to perform the beam test of detectors. This study was supported by Grant-in-Aid for JSPS Fellows and MEXT/JSPS KAKENHI Grant numbers 19654036, 19340075 and 21105004.

\section{References}

[1] T. Hatsuda and S. H. Lee, Phys. Rev. C 46, R34 (1992), T. Hatsuda, S. H. Lee and H. Shiomi, Phys. Rev. C 52, 3364 (1995).

[2] J. Brown and M. Rho, Phys. Rev. Lett. 66, 2720 (1991).

[3] F. Klingl, N. Kaiser, and W. Weise, Nucl. Phys. A 624, 527 (1997).

[4] E. Oset and A. Ramos, Nucl. Phys. A 679, 616 (2001), D. Cabrera and M. J. Vicente Vacas, Phys. Rev. C 67, 045203 (2003).

[5] M. Naruki et al.(KEK-PS E325 Collaboration), Phys. Rev. Lett. 96, 092301 (2006).

[6] R. Muto et al.(KEK-PS E325 Collaboration), Phys. Rev. Lett. 98, 042501 (2007).

[7] http://j-parc.jp/researcher/Hadron/en/pac_0606/pdf/p16-Yokkaichi_2.pdf

[8] S. Yokkaichi, Lect. Notes in Phys. 781, 161 (2009).

[9] Y. Komatsu et al., Nucl. Instr. and Meth. A 732, 241 (2013).

[10] K. Aoki et al., Nucl. Instr. and Meth. A 628, 300 (2011).

[11] S. H. Lee, Phys. Rev. C 57, 927 (1998), and Phys. Rev. C 58, 3771 (1998). 